PHANTOM C OM MUNITIES 

SCOTT DURHAM

\section{Phantom Communities}

The Simulacrum

and the Limits of

Postmodernism

STANFORD UNIVERSITY PRES

S T A N F O R D, C ALI F O R N I A

I 998 


\section{Stanford University Press}

\section{Stanford, California}

(C) 1998 by the Board of Trustees of the

Leland Stanford Junior University

Printed in the United States of America

CIP data appear at the end of the book 